\title{
Human Arm Posture Optimisation in Bilateral Teleoperation Through Interface Reconfiguration
}

\author{
Luka Peternel $^{1}$, Cheng Fang ${ }^{2}$, Marco Laghi ${ }^{3,4}$, Antonio Bicchi ${ }^{3,4}$, Nikos Tsagarakis ${ }^{3}$, and Arash Ajoudani ${ }^{3}$
}

\begin{abstract}
In this paper, we propose a method for improving the human operator's arm posture during bilateral teleoperation. The method is based on a musculoskeletal model that considers human operator's arm dynamics and the feedback force from the haptic interface (master), which is used to control a robotic arm (slave) in a remote environment. We perform an online optimisation to find the optimal configuration that has the longest endurance time with respect to muscle fatigue. Next, a trajectory is generated on the haptic interface in order to guide the human arm into the optimal configuration. The teleoperation is temporarily suspended by decoupling the master from the slave robot when the haptic device is being reconfigured. Afterwards, the loop is coupled again and the slave robot is controlled from the position where it stopped after the haptic interface guided the operator's arm to the optimised configuration. The main advantage of the proposed method is that the human operator can perform the task with less effort, which increases the endurance time. To validate our approach, we performed proof-of-concept experiments on a teleoperation system composed of two Franka Emika robots, where one was serving as master and the other as slave.
\end{abstract}

\section{INTRODUCTION}

We often strive to make robots as autonomous as possible. Nevertheless, in some cases various levels of human supervision are still required. A more direct type of supervision can be achieved through teleoperation, where the human operator handles a robotic interface (master) in order to control a remote robot (slave) that is located in a remote environment to perform a task from a distance. In a classic bilateral teleoperation, the master interface provides also a haptic feedback about the forces that are sensed on the slave robot side [1], [2] to improve the immersion of human and eventually achieve perfect telepresence [3]. In an enhanced bilateral teleoperation the human can also command the impedance of slave robot [4]-[7], which was developed from the tele-impedance concept [8].

Unlike robotic systems that can usually work under high physical loadings and may not affected for long periods of time, humans are prone to fatigue and performance degradation. While an additional force feedback improves the

\footnotetext{
${ }^{1}$ Department of Cognitive Robotics, Delft University of Technology, Mekelweg 2, 2628 CD Delft, The Netherlands, e-mail: 1.peternel@tudelft.nl

${ }^{2}$ SDU Robotics, The Maersk Mc-Kinney Moller Institute, University of Southern Denmark, Odense, Denmark

${ }^{3} \mathrm{HRI}^{2} \mathrm{Lab}, \mathrm{HCMM} \mathrm{Lab}$ and SoftBots Lab, Istituto Italiano di Tecnologia, Via Morego 30, 16163 Genoa, Italy

${ }^{4}$ Centro di Ricerca "E. Piaggio", Universita di Pisa, Largo L. Lazzarino, 1, 56126 Pisa, Italy

This work was supported in part by the European Research Council (ERC) starting grant Ergo-Lean, Grant Agreement No. 850932.
}

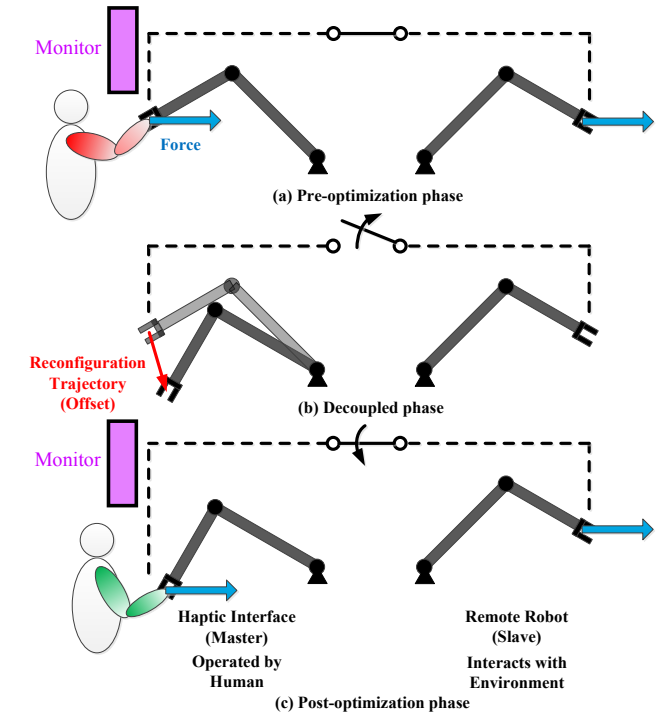

Fig. 1: Concept of the proposed method. The task force is estimated online during the teleoperation (a). Based on the estimated force and by using human models, the arm configuration is optimised by a physical movement of master haptic interface (b). After the reconfiguration phase, the teleoperation is resumed (c). The reference task force and the measured force at the remote robot are displayed to the teleoperator in real-time on a monitor. Additionally, the teleoperator can feel the feedback force through the haptic interface.

immersion of human teleoperator and can therefore improve the task performance, it also increases the effort exerted by the human arm. Being exposed to a large load can on the other hand degrade the task performance in the long run due to the physical fatigue of human muscles [9]-[11]. One solution to this problem is to employ a classic unilateral teleoperation, where the human operator commands only the motion, while the haptic interface provides no force feedback. However, the evident downside of such solution is a considerable decrease of operator's immersion due to the absence of haptic information from the remote environment. Moreover, improper postures of human teleoperator's arm, while executing various complex tasks themselves, can result in unnecessary effort and fatigue [12], [13], even if no force is fed back.

To reduce the effort and improve the ergonomics of human co-worker in human-robot collaboration settings, several methods have been recently proposed. In [14], [15] the robot physically changed the configuration of human coworker in order to reduce the effect of an external load on human joints. Some methods tried to address this issue by 
improving the manipulability of human arm in human-robot co-manipulation [15]-[17]. Other methods measured [18], [19] or modelled [20] human muscle fatigue and changed the robot's collaborative behaviour in order to reduce the effect of fatigue. While these methods have been solving the physical aspect of human ergonomics in classic human-robot collaboration settings, a similar approach is missing for the teleoperation setup.

In this paper, we introduce a novel concept for ergonomic bilateral teleoperation that physically reconfigures the human operator's arm configuration through the movement of (master) haptic interface (see Fig. 1). The system is equipped with human musculoskeletal and muscle fatigue models [20], which are used to estimate the optimal position for reconfiguration. This position maximises the fatigue-related endurance time of task production that requires exerting a specific force at the (slave) remote robot, which is in turn felt by the teleoperator through force feedback. During the reconfiguration phase, our method temporarily decouples the master robot from the slave robot and uses the master robot to change the current non-optimal working configuration of human arm into the estimated optimal configuration. In this transition stage, the slave robot configuration remains stationary. Once the reconfiguration trajectory is completed and the slave is coupled back with the master, the human operator can resume the task execution in a more ergonomic configuration. The continuity of slave robot position is guaranteed during the reconfiguration process.

The main contribution of this paper is the above-mentioned tele-operation concept for online reconfiguration of human operator's arm through the master haptic interface based on the optimal arm posture (i.e., yellow and cyan blocks in Fig. 2. However, the optimal posture is obtained by using muscle force and muscle fatigue models based on our previous work [19], [20] (i.e., red block in Fig. 2).

To demonstrate the proposed method, we perform proofof-concept experiments on bilateral teleoperation system that includes two Panda robots by Emika Franka, where one serves as a haptic interface (master) and the other serves as a remote manipulator (slave). The main task given to the operator is to produce a force on a surface with the slave robot end-effector, which can relate to common industrial tasks such as polishing and drilling. We perform an additional experiment where we induce an external payload, by putting an object with unknown mass on the slave's end-effector during the teleoperation.

\section{Methods}

The proposed method is illustrated by a block scheme in Fig. 2. First, we obtained human muscle force estimation model by offline learning using data from human musculoskeletal model. This model was then used online to estimate the individual muscle forces of human operator's arm in real time based on the measured endpoint force that was required to produce the given task (see Section II-A). We then used the estimated muscle forces in the fatigue model to estimate the endurance times in different working

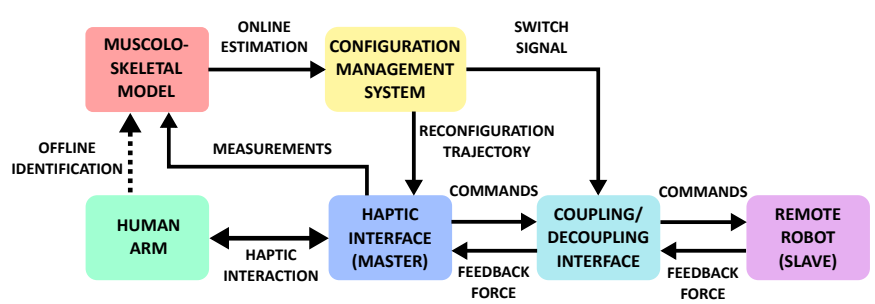

Fig. 2: Block scheme of the proposed method. A bilateral teleoperation system is enhanced by a human arm configuration management system. The human muscle force is estimated by the musculoskeletal model (red), which includes the muscle fatigue model to estimate the fatigue of individual muscles. The management system (yellow block) performs an online optimisation to optimise the teleoperator's arm configuration, in which the task can be produced with the least amount of fatigue. When the optimal configuration is obtained, the slave robot is decoupled from the master interface and the master is used to guide the operator's arm to that configuration. After online reconfiguration phase, the slave is coupled back into the teleoperation loop and the task execution is resumed.

configurations within the selected workspace (see Section IIB. The optimisation algorithm then searched for the optimal configuration, where the endurance time is maximised.

Initially the operator accomplished the task by controlling the remote slave robot through the bilateral teleoperation. We used $\mathrm{FT}^{2}$ bilateral teleoperation architecture from [7], which assured the stability and transparency requirements in [1], [6]. To show the adaptability of our approach, we assumed that the task is not know in advance. Therefore, the operator first needed to produce the task for a while before the online optimisation could be performed under the measured conditions. When the task parameters were measured and were known to the human arm configuration management system, the online optimisation took place and the slave robot was temporarily decoupled from the teleoperation loop. In the meantime the arm configuration management system generated a trajectory that moved the master interface in a way that the optimal human arm configuration was obtained (see Section II-C).

It is important to note that before the master is decoupled, the protocol dictates that the human operator stops producing any force or motion with the slave in order to ensure safety and stability during the transitional decoupling phase. It also ensures that there is no force or motion when the slave is coupled back into the loop. If any physical interaction or motion is detected, the system postpones the decoupling and reconfiguration until the operator stops producing force and motion on both master and slave side.

When the human arm was moved into the optimal configuration and was ready to resume the desired task, the slave robot was coupled back with the master interface to again form a bilateral teleoperation. Since the slave remained stationary during the reconfiguration, an extra position offset between the master robot positions before and after the reconfiguration process was added to the commanded position 1 . The operator then resumed performing the same task as before in a more ergonomic condition.

\footnotetext{
${ }^{1}$ This is similar to the methods that induce an offset in commanded position to span the limited kinematic workspace of master device [21]. In contract, we use the offset to improve operator's ergonomics.
} 


\section{A. Human Arm Muscle Force Model}

In quasi-static conditions, the relationship between the force at the endpoint of human arm that comes from interaction with the haptic interface (master) and the joint torques in the human arm is defined as

$$
\boldsymbol{\tau}=\boldsymbol{J}^{T}(\boldsymbol{q}) \boldsymbol{f}+\boldsymbol{g}(\boldsymbol{q}),
$$

where $f$ is the endpoint force vector, $\tau$ is the joint torque vector, $\boldsymbol{J}$ is the human arm geometric Jacobian matrix, $\boldsymbol{q}$ is the arm joint configuration and $\boldsymbol{g}$ is the torque vector arising from the arm's gravity. Note that the endpoint force $f$ is caused by the interaction between the operator's arm and the haptic interface, and is equal to the force that the slave robot produces in the remote environment. The degrees of freedom (DoF) of human arm model depend on the model complexity. The general model has seven main DoF in joint space $\left(\boldsymbol{q}, \boldsymbol{\tau}, \boldsymbol{g} \in \mathbb{R}^{7}\right)$ and six DoF in Cartesian space $(\boldsymbol{f} \in$ $\mathbb{R}^{6}$ ), nevertheless in the experiments we considered a planar 2D case.

To produce the desired joint torques, the muscles in the arm have to produce muscle forces, where the relationship is given as

$$
\boldsymbol{\tau}=\boldsymbol{J}_{t}^{T}(\boldsymbol{q}) \boldsymbol{f}_{t}
$$

where $\boldsymbol{f}_{t}$ is the muscle force vector and $\boldsymbol{J}_{t}$ is the muscle Jacobian matrix composed of muscle tendon moment arms.

By combining (1) and (2) we derive to the relationship between the human arm muscle forces and the endpoint force, which is defined as

$$
\boldsymbol{f}_{t}=\boldsymbol{J}_{t}^{-T}(\boldsymbol{q})\left(\boldsymbol{J}^{T}(\boldsymbol{q}) \boldsymbol{f}+\boldsymbol{g}(\boldsymbol{q})\right) .
$$

Note that the transformation $\boldsymbol{J}_{t}^{-T}$ has a redundancy, since there is typically more than one muscle acting on a single joint. This produces a large amount of possible solutions, many of which are practically unfeasible.

Literature addressed this kind of redundancy resolution by employing optimisation techniques that can find some physiologically most feasible solution. Some of these methods include: Static Optimisation (SO) [22], Computed Muscle Control [23] and Neuromusculoskeletal Tracking [24]. In this work we employed SO because of its computational robustness and efficiency.

The optimisation problem of distributing muscle forces in SO can be formulated as

$$
\text { s.t. } \underset{\boldsymbol{J}_{t}^{T}}{\underset{\boldsymbol{a}}{\arg \min \boldsymbol{a}^{T} \boldsymbol{a}}}(\underbrace{\boldsymbol{F}_{t}^{0} \boldsymbol{a}}_{\boldsymbol{f}_{t}})=\boldsymbol{\tau},
$$

where $\boldsymbol{a} \in\left[\begin{array}{ll}0 & 1\end{array}\right]$ indicates the muscle activation vector and $\boldsymbol{F}_{t}^{0}$ is a constant diagonal matrix, the elements of which denote the maximum isometric forces of the corresponding muscles. The muscle force $\boldsymbol{f}_{t}$ is then defined as a product of $\boldsymbol{F}_{t}^{0}$ and $\boldsymbol{a}$, implying that muscle is considered as an ideal force generator in SO. It is apparent that by holding the constraint of (2), this optimisation tries to minimise the sum of squares of muscle activations, which is a commonly employed objective function in the related research [25].

In the offline phase we used the above-mentioned method to estimate muscle forces for a variety of endpoint forces throughout the human arm workspace. This data was then stored for later use in online optimisation of operators arm configuration for the given task force.

\section{B. Human Muscle Fatigue Model}

We used the muscle forces from the musculoskeletal model to estimate the individual muscle fatigue in the selected human arm workspace, given the external interaction force produced by the haptic interface. To estimate the muscle fatigue, we used the model that we recently proposed in [20], which is defined as a first-order system of differential equations

$$
\frac{d V_{i}(t)}{d t}=\left\{\begin{array}{ll}
\left(1-V_{i}(t)\right) \frac{f_{t i}(t)}{C_{i}} & \text { if } f_{t i}(t) \geq f_{t h} \\
-V_{i}(t) \frac{R}{C_{i}} & \text { if } f_{t i}(t)<f_{t h}
\end{array},\right.
$$

where $V_{i} \in\left[\begin{array}{ll}0 & 1\end{array}\right]$ denotes the muscle fatigue level of the $i$ th muscle, $f_{t i}$ is the $i$-th muscle force as obtained from (4) and $C_{i}$ represents the capacity parameter of the $i$-th muscle. The parameters $C$ define the characteristics of fatigue and are dependent on individual human operator and muscles. For example, if the parameter $C$ is high, the muscle will get tired slowly under a given effort $f_{t}$, compared to when $C$ is low.

To determine the parameters $C$, we initially performed a calibration procedure as proposed in [19]. In this procedure the human had to exert several reference muscle forces $f_{t}^{r e f}$ for a period of time $T_{\text {end }}$, which indicated a limit when the given reference could not be endured anymore, or beyond which it became uncomfortable to do so. The parameter $C$ was then obtained for each reference force $f_{t}^{r e f}$ as

$$
C=-\frac{f_{t}^{r e f} \cdot T_{\text {end }}}{\log (1-0.993)},
$$

where we assumed that the full capacity is reached at $V=$ 0.993 , which corresponds to five time constants. The final estimation of fatigue capacity $C$ was obtained by the mean of $C$ parameters for different tested reference forces of the same muscle.

The recovery part of the model (i.e., the lower part of (5p) includes the parameter $R$, which defines how fast the fatigue will dissipate when the given muscle is relaxed. The relaxed state of the muscle is defined by the threshold $f_{t h}$. If $f_{t}$ is larger than $f_{t h}$, the muscle is in fatigue mode, otherwise it is in relaxed mode. In these proof-of-concept experiments the task involved prolonged effort, therefore the relaxed mode was never activated. However, we keep the full definition of the model for generality.

\section{Configuration Management and Decoupling System}

The data from the fatigue model was used in an online optimisation process that estimated the human operator's arm configuration, in which the task could be produced with the longer endurance time. The optimal configuration 


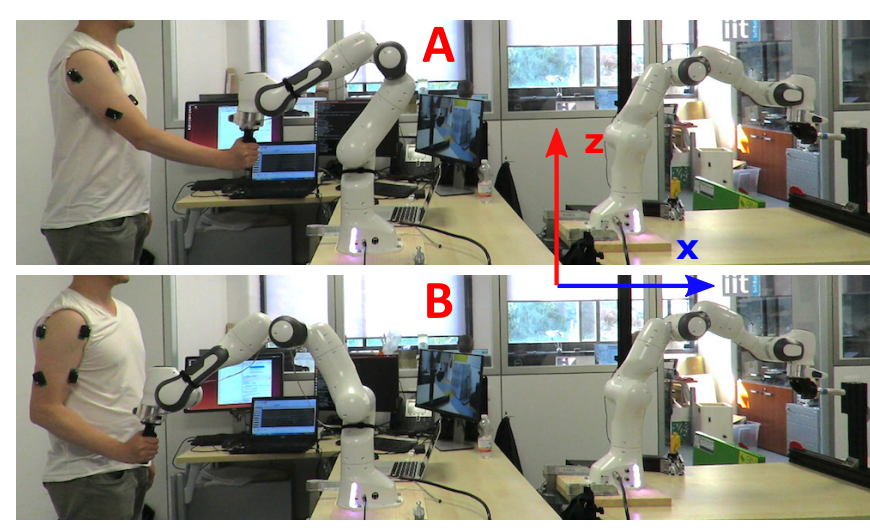

Fig. 3: Experimental setup for force production task scenario in teleoperation. A reconfiguration of master robot (on the left-hand side) is planned and implemented during the teleoperation based on an optimisation of the ergonomics of human operator's arm. The slave robot (on the right-hand side) keeps its configuration during the reconfiguration of master robot. The photo A was taken during the experiment before the reconfiguration phase. The photo B was taken after the reconfiguration phase.

is dependent on a given interaction force with the haptic interface (dictated by the task produced at the slave robot side), human arm dynamic parameters and muscle fatigue parameters. By using the muscle fatigue model as defined in (5), the maximum endurance time for each muscle can be derived as

$$
T_{i}(\boldsymbol{q}, \boldsymbol{f})=-\frac{C}{f_{t i}(\boldsymbol{q}, \boldsymbol{f})} \ln \left(1-V_{t h}\right),
$$

where the endpoint interaction force $f$ is defined by the task and $V_{t h}$ is the fatigue threshold for the given muscle, above which we do not want to exceed. Note that this threshold can be tuned individually for each muscle based on the desired conditions and safety specifications. The optimisation process in (4) was used to get the individual muscle forces $f_{t}(\boldsymbol{q}, \boldsymbol{f})$.

Different configurations of the arm give different relationships between muscle forces and endpoint force, as well as different gravity torques (see (3)), and therefore the arm would have different endurance times for the same given endpoint task force in different configurations. The overall arm endurance time in any given configuration is equal to the minimum of endurance times of all considered muscles $i$ in that configuration. The optimal position of the arm endpoint can be found by maximising the overall arm endurance time in all possible configurations within the selected workspace, and can be formally defined as

$$
\underset{\boldsymbol{q}}{\operatorname{argmax}}\left(\min _{i}\left(T_{i}(\boldsymbol{q}, \boldsymbol{f})\right)\right),
$$

where index $i$ corresponds to the individual muscle of human arm, as before. In this optimisation process we also considered the constraints of $\boldsymbol{q}$ that comes from the joint limits and the selected endpoint workspace. The desired task dictates the force $f$ and should also be constrained accordingly. We used C++ ALGLIB library to solve (8).

After the optimal configuration was obtained from 87 for the desired task force $f$, we decoupled the remote robot (slave) from teleoperation loop and switched the control mode on the haptic interface (master). The coupled and decoupled control modes are conceptually defined as

$$
\begin{gathered}
\boldsymbol{x}_{s}(t)=\left\{\begin{array}{ll}
\boldsymbol{x}_{m}(t)-\boldsymbol{x}_{\text {offset }} & \text { if mode }=\text { coupled } \\
\boldsymbol{x}_{m}\left(t_{d}\right) & \text { if mode }=\text { decoupled }
\end{array},\right. \\
\boldsymbol{f}_{m}(t)=\left\{\begin{array}{ll}
\boldsymbol{f}_{s}(t) & \text { if mode }=\text { coupled } \\
\boldsymbol{f}_{r}(\delta) & \text { if mode }=\text { decoupled }
\end{array},\right.
\end{gathered}
$$

where $\boldsymbol{x}_{s}$ and $\boldsymbol{x}_{m}$ are slave and master positions, respectively, while $\boldsymbol{f}_{s}$ and $\boldsymbol{f}_{m}$ are slave and master forces, respectively. Master offset position $\boldsymbol{x}_{\text {offset }}$ was initially zero and was then changed after the reconfiguration phase to account for the new arm configuration of operator. Note that decoupling mode can be entered only when the human operator stops producing any force or motion with the slave in order to ensure safety and stability during the transition. When decoupling happened, the slave position $\boldsymbol{x}_{s}$ remained at its last position at the time of decoupling $t_{d}$ for duration of reconfiguration phase ( $\delta$ from 0 to $t_{\delta}$ ), while master force $\boldsymbol{f}_{m}$ was used by the impedance controller to perform the reconfiguration trajectory as

$$
\boldsymbol{f}_{r}(\delta)=\boldsymbol{K}\left(\boldsymbol{x}_{r}(\delta)-\boldsymbol{x}_{m}(t)\right)+\boldsymbol{D}_{r}\left(\dot{\boldsymbol{x}}_{r}(\delta)-\dot{\boldsymbol{x}}_{m}(t)\right),
$$

where $f_{r}$ is the end-effector at the master used during the reconfiguration in (10), $\boldsymbol{x}_{r}$ and $\dot{\boldsymbol{x}}_{r}$ are actual and desired end-effector position and velocity of reconfiguration trajectory, respectively, while $\boldsymbol{K}$ is Cartesian stiffness and $\boldsymbol{D}$ is Cartesian damping matrix.

We generated the reconfiguration trajectory $\boldsymbol{x}_{r}$ between the current task execution position and the new optimal position by a fifth-order polynomial method. In the end, the master executed the generated trajectory and moved the human arm into the new configuration. After the trajectory was executed on the master side, we coupled the slave back with the master, and the human operator resumed the teleoperation. The commanded slave position was then equal to the new master position $\boldsymbol{x}_{m}(t)$ plus an offset with respect to the previous configuration $\boldsymbol{x}_{\text {offset }}$ (see (9) that was generated by the reconfiguration trajectory. This process was triggered and executed automatically by the system.

\section{EXPERIMENTS}

The main experiments involved a task where an experienced human operator had to produce a force on an object in a remote environment by teleoperating the slave robot. Fig. 3 shows an illustration of the experimental setup for the force production task. We selected two force references during the experiments. The first reference was defined by producing a force of $20 \mathrm{~N}$ in positive $\mathrm{x}$-axis, while the second reference was defined by producing a force of $20 \mathrm{~N}$ in negative $\mathrm{z}$ axis. The reference was displayed to the teleoperator on a screen in real-time. A supplementary experiment involved external payload at the slave robot, where we put an object with mass of $2 \mathrm{~kg}$ to its end-effector during the teleoperation. Note that the mass of the object was unknown to the human operator and the system. Fig. 4 shows an illustration of 


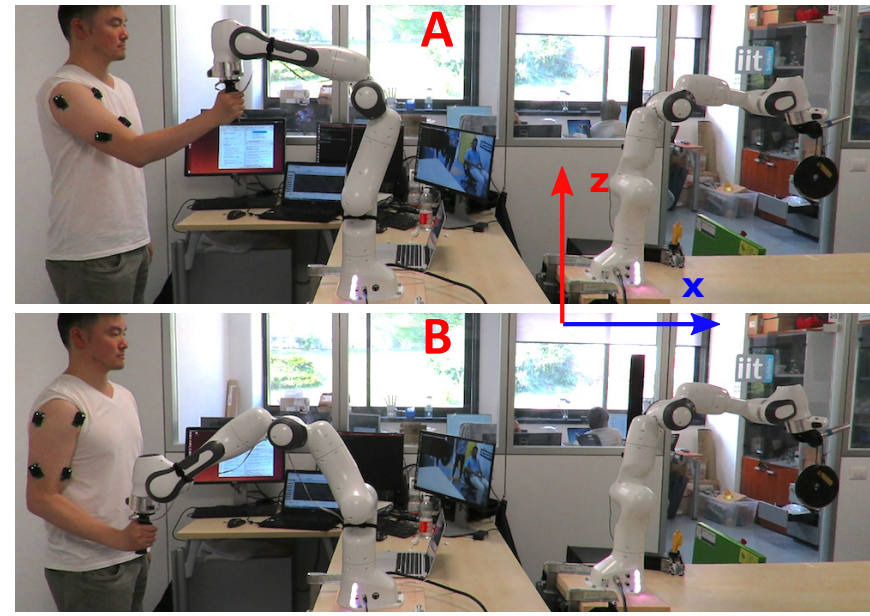

Fig. 4: Experimental setup for external payload scenario in teleoperation. An optimisation-based reconfiguration of master robot, similar to the one used in the force production task scenario shown in Fig. 3 is planned and implemented. The photo $\mathrm{A}$ was taken during the experiment before the reconfiguration phase. The photo $\mathrm{B}$ was taken after the reconfiguration phase.

the experimental setup for the external payload scenario. The goal of the proposed method in both experiments was to determine the current task parameters and optimise the human operator's arm configuration at the master side in order to maximise the endurance time related to the muscle fatigue.

In order to be able to change the configuration of human arm, the master haptic interface has to have a feedback about the current operator's arm configuration. In these proof-ofconcept experiments, we considered optimisation only in sagittal (x-z) plane, however the method is not limited to a plane. Since the experiments were limited to sagittal plane, the joint configuration could be calculated in real-time based on the kinematic model of the arm and the current endpoint position, which was measured by the haptic interface. In case of 3D space analysis with redundant DoF, the joint positions can be measured by optical motion capture system or robot vision.

\section{A. Force Production Task}

In the optimisation process of these proof-of-concept experiments we considered four arm muscles that have dominant roles in arm endpoint force production: Anterior Deltoid (AD), Posterior Deltoid (PD), Biceps Brachii (BB) and Triceps Brachii (TB). The former two are involved in the shoulder motion, while the latter two are primarily involved in the elbow motion, with some contribution to the shoulder motion as well. Note that the proposed method can consider arbitrary number of muscles.

We performed a preliminary experiment to determine the parameters related to the muscle fatigue model. For additional validation, we also measured surface electromyography (EMG) of the considered muscles using Delsys Trigno system (sampling rate $1000 \mathrm{~Hz}$ ). In a preliminary calibration experiment, we measured EMG at maximal voluntary contraction (MVC) in order to normalise EMG signals and

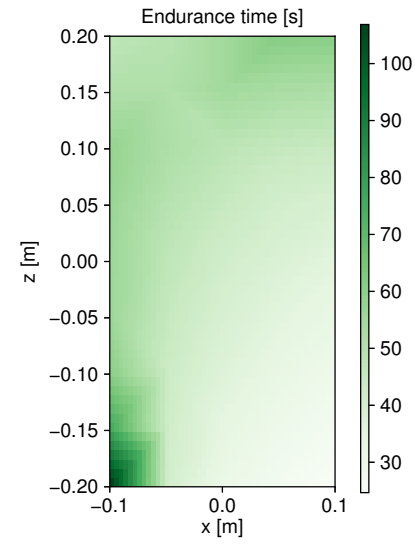

(a) plus $x$-axis

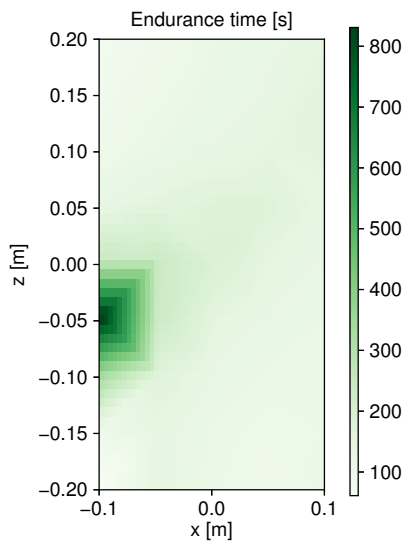

(b) minus z-axis
Fig. 5: Results of teleoperator's arm position optimisation process for force production tasks. The graph on the left shows muscle fatigue-based endurance times (in seconds) for producing the endpoint force of $20 \mathrm{~N}$ in positive $\mathrm{x}$-axis in different positions of the selected arm workspace. The graph on the right shows the same for the endpoint force of $20 \mathrm{~N}$ in negative $\mathrm{z}$-axis. The position of arm endpoint $(0,0)$ corresponds to configuration when the upper arm is aligned along the body and the forearm is rotated upward by $90^{\circ}$. The optimal arm configuration that corresponds to the left graph is shown in photo B of Fig. 3
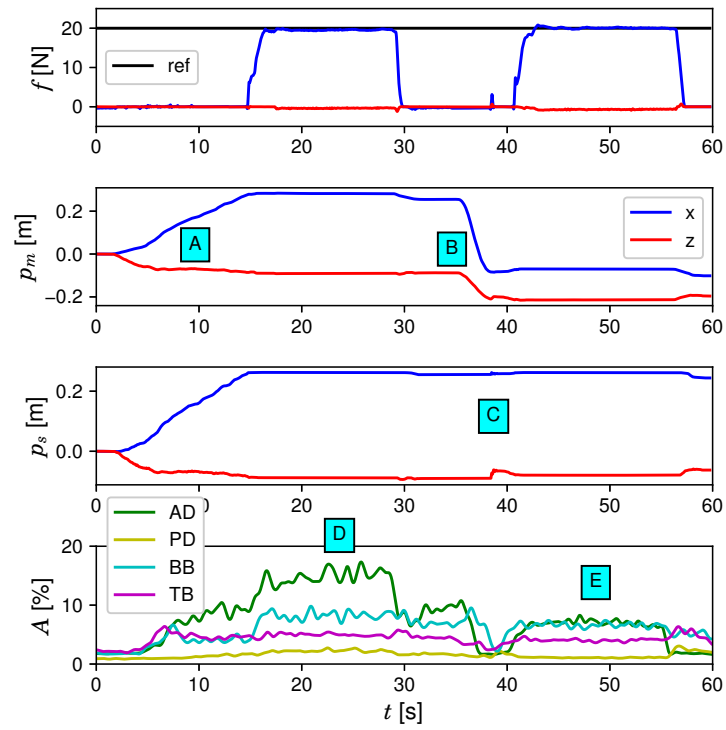

Fig. 6: Results of the force production experiment. The first graph shows the force at the slave robot end-effector, were the black line is the reference force, the blue line is $\mathrm{x}$-axis force and the red line is $\mathrm{z}$-axis force. The second graph shows the position of human arm endpoint and haptic interface (master). The third graph shows the position of slave robot end-effector. The fourth graph shows muscle activations as measured by EMG.

obtain muscle activations $A$. Note that EMG is not necessary for functioning of the proposed method, but it was only used for extra validation.

The results of human arm configuration optimisation for the main task, as described by (8), are shown in Fig. 5 . We can see how the muscle fatigue-related endurance time for the given task force is affected by different endpoint positions of human arm. It can also be observed that the optimal position changes with the direction of the endpoint force (comparison between left and right graphs), due to the changed involvement of the considered arm muscles. For the 


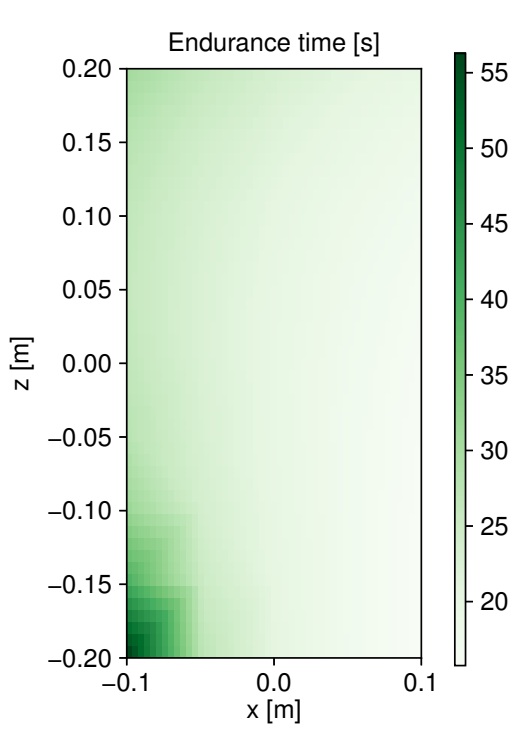

(a) optimisation
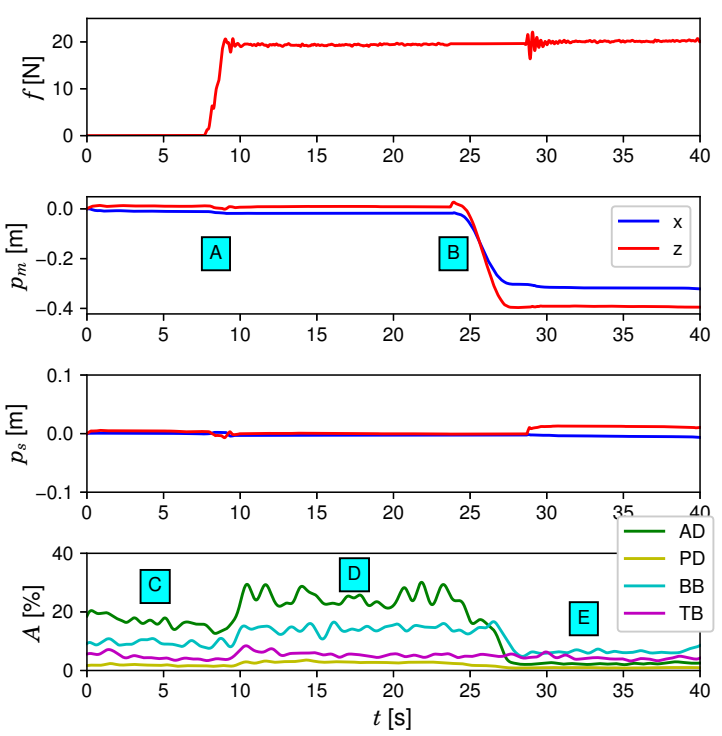

(b) experiment

Fig. 7: Results of the external payload experiment. The graph on the left shows muscle fatigue-based endurance times (in seconds) for producing the object gravity-compensation force in positive z-axis (i.e., opposite of gravity) in different positions of the selected arm workspace. The optimal arm configuration that corresponds to the left graph is also shown in photo B of Fig. 4 The graphs on the right show the measured slave force in z-axis, master position, slave position and muscle activations, respectively, in a similar manner as in Fig. 6

reference force production in positive $\mathrm{x}$-axis, the optimisation prescribed optimal endpoint position at $(\mathrm{x}, \mathrm{z})=(-0.1,-0.2)$. See also photo B in Fig. 3 for an illustration of the optimal human arm configuration during the experiment. On the other hand, the optimal endpoint position for the reference force production in negative $\mathrm{z}$-axis was located at $(\mathrm{x}, \mathrm{z})=(-0.1$, $0.05)$. The positions are given with respect to the initial arm configuration, where the upper arm was aligned along the body and the forearm was rotated upward by $90^{\circ}$.

It should be noted that the global optimum is usually found at a singular configuration of arm, when it is aligned with the body (i.e., when effect of gravity is minimised). However, since we selected this particular desired workspace, the optimisation searched for an optimum within the selected workspace (see Fig. 5 and Fig. 6 for the optima under two different conditions within the workspace). Different desired workspace can be freely selected by the user.

The endurance time for producing force in negative $z$ axis in the given optimal configuration was considerably higher (862.4 seconds) compared to the endurance time for producing force in positive $\mathrm{x}$-axis (106.9 seconds), even though the force magnitude was the same (i.e., $20 \mathrm{~N}$ ). This comes from the fact that the force production in negative $\mathrm{z}$-axis is assisted by the force of gravity and therefore much less muscular effort is required.

The results of the experiment for the task, where the force had to be produced on the object surface in positive x-axis, are shown in Fig. 6. In the second graph, we can see how the human operator first moved the slave robot near the object (see the section marked by letter A in the graph), on which it had to produce the task force. The slave robot position followed accordingly, as it can be observed in the third graph. When the contact with the object was established, the human operator increased the force in $\mathrm{x}$-axis to achieve the desired reference force. The proposed system measured the task force and performed the optimisation process to find the optimal human arm position, where the fatigue-related endurance time was maximised. The system then planned a trajectory that could move the human arm into that position. At that point, the slave robot was decoupled from the master, and the haptic interface moved the human arm according to the generated trajectory. This trajectory can be observed around the section marked by B in the graph. Note that the slave robot position remained still, while the master position changed.

After the haptic interface successfully performed the reconfiguration of human arm, master and slave were coupled again into the teleoperation loop. This event is marked by $\mathrm{C}$ in the graph. After the teleoperation was resumed from the unchanged slave robot position, the human operator continued to produce the task force on the object in x-axis.

For additional validation we can also see the human operator's arm muscle activity in the fourth graph of Fig. 6 . It is evident that the muscle activity was in general higher in the initial position (see the section marked by D), compared to the optimised position (see the section marked by $\mathrm{E}$ ).

\section{B. External Payload Task}

The results of supplementary experiment that involved external payload, where we put an object on the slave's end-effector, are shown in Fig. 77. The optimisation process regarding the fatigue-based endurance time is shown on the left graph, where the optimal endpoint position to hold the object was at $(\mathrm{x}, \mathrm{z})=(-0.1,-0.2)$. The graphs on the right side show the course of the experiment. The object was put on the slave at the point marked by letter A. After that, we can see that the measured force in $\mathrm{Z}$-axis was increased (top 
graph). Consequently, also the muscle activity of operator's arm increased (see the difference between section marked by $\mathrm{C}$ and $\mathrm{D}$ in the bottom graph). When the system determined the force of additional payload and optimised the master side position, the slave was decoupled and the master moved the human into the optimal position (see the section marked by B in the second graph). After the reconfiguration, the slave was coupled again with the master into the teleoperation loop. We can see a significant decrease of measured muscle activity in the operator's arm (notice the difference between section marked by $\mathrm{D}$ and $\mathrm{E}$ in the bottom graph).

\section{Discussion}

The main advantage of the proposed method is that it can provide a more ergonomic working condition for the human operator in the teleoperation scenario. Using the human arm muscle force estimation model and muscle fatigue model, the online optimisation process can find the optimal arm position to produce the given task with maximum endurance time. The experimental results showed that the EMG-based measured muscle activity of human operator's arm significantly decreased after the proposed method performed the online reconfiguration. This supports the increased endurance time calculated by the muscle fatigue model.

One potential drawback of the proposed method is that it relies on human musculoskeletal and fatigue models. The precision of optimisation is therefore limited to the precision of the models that are used. In addition, some calibration procedures are initially required to identify model parameters, which can be time-consuming. Nevertheless, if teleoperator's body properties remain relatively constant, the identified parameters can be used for extended period of time.

Another potential limitation is that classic haptic interfaces can only influence the human arm configuration through endpoint and cannot directly change joint positions, if there are redundant DoF. Such limitation can be solved by an additional visual feedback that can account also for nullspace motion of human arm in joint space.

In future, we will include other ergonomic metrics into the proposed framework, such as muscle manipulability [17]. We will also consider experimental tasks that include periods of rest, where the recovery mode of fatigue model should be considered (i.e., the lower part of (5)).

\section{REFERENCES}

[1] D. A. Lawrence, "Stability and transparency in bilateral teleoperation," IEEE Transactions on Robotics and Automation, vol. 9, no. 5, pp. 624637, Oct 1993.

[2] K. J. Kuchenbecker and G. Niemeyer, "Induced master motion in force-reflecting teleoperation," Journal of Dynamic Systems, Measurement, and Controlh, vol. 128, no. 4, pp. 800 - 810, April 2006.

[3] R. M. Held and N. I. Durlach, "Telepresence," Presence: Teleoperators \& Virtual Environments, vol. 1, no. 1, pp. 109-112, 1992.

[4] L. Peternel, T. Petrič, and J. Babič, "Human-in-the-loop approach for teaching robot assembly tasks using impedance control interface," in Robotics and Automation (ICRA), 2015 IEEE Intl. Conf. on, May 2015, pp. 1497-1502.

[5] L. Peternel, T. Petrič, and J. Babič, "Robotic assembly solution by human-in-the-loop teaching method based on real-time stiffness modulation," Autonomous Robots, vol. 42, no. 1, pp. 1-17, Jan 2018.
[6] M. Laghi, A. Ajoudani, M. Catalano, and A. Bicchi, "Tele-impedance with force feedback under communication time delay," in 2017 IEEE/RSJ International Conference on Intelligent Robots and Systems (IROS), Sept 2017, pp. 2564-2571.

[7] M. Laghi, A. Ajoudani, M. G. Catalano, and A. Bicchi, "Unifying bilateral teleoperation and tele-impedance for enhanced user experience," The International Journal of Robotics Research, vol. 39, no. 4, pp. 514-539, 2020.

[8] A. Ajoudani, N. G. Tsagarakis, and A. Bicchi, "Tele-impedance: Teleoperation with impedance regulation using a body-machine interface," The International Journal of Robotics Research, vol. 31, no. 13, pp. 1642-1656, 2012.

[9] C. J. De Luca, "Myoelectrical manifestations of localized muscular fatigue in humans." Critical reviews in biomedical engineering, vol. 11, no. 4, pp. 251-279, 1984.

[10] R. M. Enoka and J. Duchateau, "Muscle fatigue: what, why and how it influences muscle function," The Journal of physiology, vol. 586, no. 1, pp. 11-23, 2008.

[11] L. Ma, D. Chablat, F. Bennis, and W. Zhang, "A new simple dynamic muscle fatigue model and its validation," Intl. Journal of Industrial Ergonomics, vol. 39, no. 1, pp. 211 - 220, 2009.

[12] E. H. Lawson, M. J. Curet, B. R. Sanchez, R. Schuster, and R. Berguer, "Postural ergonomics during robotic and laparoscopic gastric bypass surgery: a pilot project," Journal of robotic surgery, vol. 1, no. 1, pp. 61-67, 2007.

[13] T. Lin, A. U. Krishnan, and Z. Li, "Physical fatigue analysis of assistive robot teleoperation via whole-body motion mapping," in 2019 IEEE/RSJ International Conference on Intelligent Robots and Systems (IROS), Nov 2019, pp. 2240-2245.

[14] W. Kim, J. Lee, L. Peternel, N. Tsagarakis, and A. Ajoudani, "Anticipatory robot assistance for the prevention of human static joint overloading in human-robot collaboration," IEEE Robotics and Automation Letters, vol. 3, no. 1, pp. 68-75, 2018.

[15] L. Peternel, W. Kim, J. Babič, and A. Ajoudani, "Towards ergonomic control of human-robot co-manipulation and handover," in 2017 IEEE-RAS 17th International Conference on Humanoid Robotics (Humanoids), Nov 2017, pp. 55-60.

[16] S. Gopinathan, S. K. Ötting, and J. J. Steil, "A user study on personalized stiffness control and task specificity in physical human-robot interaction," Frontiers in Robotics and AI, vol. 4, p. 58, 2017.

[17] T. Petrič, L. Peternel, J. Morimoto, and J. Babič, "Assistive armexoskeleton control based on human muscular manipulability," Frontiers in Neurorobotics, vol. 13, p. 30, 2019.

[18] B. Sadrfaridpour, H. Saeidi, J. Burke, K. Madathil, and Y. Wang, Modeling and Control of Trust in Human-Robot Collaborative Manufacturing. Boston, MA: Springer US, 2016, pp. 115-141.

[19] L. Peternel, N. Tsagarakis, D. Caldwell, and A. Ajoudani, "Robot adaptation to human physical fatigue in human-robot comanipulation," Autonomous Robots, vol. 42, no. 5, pp. 1011-1021, Jun 2018.

[20] L. Peternel, C. Fang, N. Tsagarakis, and A. Ajoudani, "A selective muscle fatigue management approach to ergonomic human-robot co-manipulation," Robotics and Computer-Integrated Manufacturing, vol. 58, pp. $69-79,2019$.

[21] F. Conti and O. Khatib, "Spanning large workspaces using small haptic devices," in World Haptics Conference, March 2005, pp. 183-188.

[22] A. Erdemir, S. McLean, W. Herzog, and A. J. van den Bogert, "Modelbased estimation of muscle forces exerted during movements," Clinical biomechanics, vol. 22, no. 2, pp. 131-154, 2007.

[23] D. G. Thelen, F. C. Anderson, and S. L. Delp, "Generating dynamic simulations of movement using computed muscle control," Journal of biomechanics, vol. 36, no. 3, pp. 321-328, 2003.

[24] A. Seth and M. G. Pandy, "A neuromusculoskeletal tracking method for estimating individual muscle forces in human movement," Journal of biomechanics, vol. 40, no. 2, pp. 356-366, 2007.

[25] F. C. Anderson and M. G. Pandy, "Static and dynamic optimization solutions for gait are practically equivalent," Journal of biomechanics, vol. 34, no. 2, pp. 153-161, 2001. 